\title{
repair in rheumatics: Is it still worthwhile?
}

\section{Manuel J. Antunes}

University Hospital, Coimbra, Portugal

Address for correspondence:

Prof Manuel J Antunes

Centro de Cirurgia Cardiotorácica

Hospitais da Universidade

3000-075 Coimbra

Portugal

Email:

antunes.cct.huc@sapo.pt

Prosthetic valve replacement is plagued by several types of complications which carry high mortality and morbidity. Among these, thromboembolism is the most feared. Thrombosis of mechanical prosthesis, especially, is a very lethal complication with a mortality rate of up to $60 \%$ and is more frequent in the mitral valve position. (1) On the other hand, degeneration of bioprostheses limits durability and requires multiple reoperations, especially in younger patients. In addition, the mortality rate of reoperations is higher than that of primary surgery. It varies with the pathology, the condition of the patient and the experience of the surgical team, but ranges from $3 \%$ to $10 \%$ in most reports. ${ }^{(2,3)}$

All these complications are even more frequent in rheumatic populations, characterised by a low mean age and poor socioeconomical conditions, which makes adherence to anticoagulation

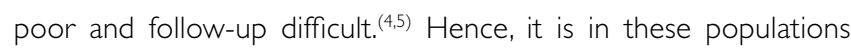
where the alternative of valve repair, especially of the mitral valve, would have the greatest benefit. Unfortunately, the results of mitral valvuloplasty in rheumatic mitral regurgitation are not as good as those observed in degenerative mitral regurgitation. But, how do these compare with those of prosthetic valve replacement in the same population? In my own experience at the Baragwanath

\section{ABSTRACT}

Prosthetic valve replacement in young patients carries increased morbidity and mortality, even with recent types and models of prostheses. Fortunately, rheumatic mitral regurgitation in this young population group is amenable to repair, although the results are less favourable than those observed with other types of mitral valve disease and in older populations. A better knowledge of the pathology and evolution of repair techniques has improved results. Hence mitral valve repair is still worthwhile, even in rheumatic pathology and, the percentage of valves repaired, increases with the experience and the will of the surgeon to preserve the valve. Mitral valve replacement can only be justified when good repair is not feasible. It is vital that the surgeon has adequate experience which can only be gained by exposure to enough patients with this condition. Most of these patients are in developing countries and hampered by socio-economic conditions - which means Ist World surgeons get limited required exposure. SAHeart 20 I0; 7:258-263

Hospital, patients who had mitral valve repair lived longer than those who had valve replacement and freedom from reoperation was similar (Figure I).(6) One further advantage of valvuloplasty is that, if and when reoperation is needed, it can usually be performed with mortality similar to that of primary surgery.

These considerations usually do not pertain to the other form of rheumatic pathology, mitral stenosis. In these cases, when the valve is pliable, valvuloplasty can be performed with excellent long term results. Initially it was done by surgical closed commissurotomy, performed digitally or with a Tubbs dilator. Since then, this procedure has mostly been substituted by open mitral commissurotomy whereby the fused commissures can be visually opened with a scalpel, concomitantly intervening on the sub-mitral apparatus, if required. The closed procedure is however still performed in many developing countries with excellent results because of the advantage of low cost. 
The surgical procedures were recently almost completely replaced by balloon mitral valvulotomy of which the main advantage is the avoidance of sternotomy and cardiopulmonary bypass. In my view, the results of this procedure are not as good as those of open mitral commissurotomy. In a series of 100 cases of open mitral commissurotomy performed in my department, systematically analysed by serial echocardiography, the mitral valve area was $2.94 \mathrm{~cm}^{2}$ immediately after surgery and $2.37 \mathrm{~cm}^{2}$ at ten-year followup. (7) These values are significantly superior to those commonly reported after balloon commissurotomy, which vary, in most series, from 2.0 to $2.2 \mathrm{~cm}^{2}{ }^{(8)}$ In the past twenty years, we have performed open mitral commissurotomy in well over I 500 cases. Obviously, not all valves are pliable and a number of them require valve replacement, but the surgery can still save many such valves.

For the purpose of this article I will, from now on restrict my comments to mitral regurgitation. One needs to distinguish between rheumatic mitral regurgitation presented in patients from developed countries in Europe and Asia and that of patients in developing countries.

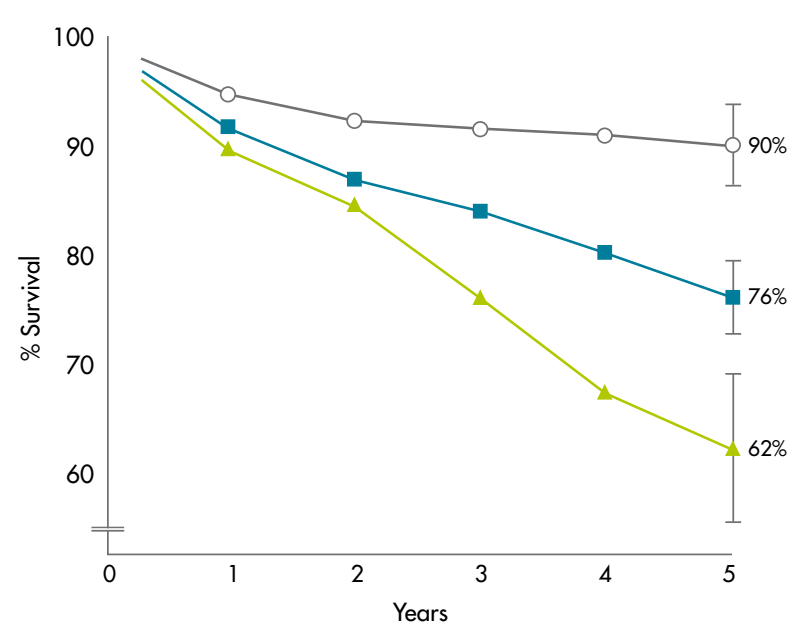

Valvuloplasty $\square$ MVR-MEC $\triangle M V R-B I O$

FIGURE I: Actuarial survival of young patients subjected to mitral valve surgery for rheumatic disease. Survival was better after valvuloplasty, as compared to replacement with a mechanical valve (MEC) or a bioprosthesis $(\mathrm{BIO}){ }^{(6)}$
Patients in developed countries are usually older mostly in their fifties or sixties. They have a preponderance for annular dilation most often with good leaflet tissue without prolapse - hence relatively easy to repair, provided that the mitral apparatus is not significantly fibrotic; and, as a result, have excellent long term outcomes. In a recent report, Wang et al. demonstrated a 6-year survival of approximately $80 \%{ }^{\left({ }^{9}\right)}$

By contrast, patients seen in developing countries are young mostly between 20 and 30 years of age. The diseased mitral valve is characterised by: Prolapse of the anterior leaflet; retraction of the posterior leaflet; annular dilatation; and, above all, relatively short leaflet tissue, thus rendering repair difficult. Moreover many patients present to surgery in the acute phase of rheumatic carditis.

\section{TECHNIQUE OF MITRAL VALVE REPAIR}

The mitral apparatus is a complex structure. It is composed of leaflets, chordae tendineae and papillary muscles, and even the ventricular and atrial walls play a role in its function. The nature of the rheumatic pathology of the mitral valve, with surgical implications, was largely not understood until the works of Carpentier in the seventies and eighties. ${ }^{(10)}$ It has since clearly been demonstrated that in rheumatic regurgitation of the young patients there is most frequently elongation or rupture of the chordae tendineae of the anterior leaflet, causing prolapse in about $90 \%$ of the cases in my experience. By contrast, the chordae tendineae of the posterior leaflet are usually normal, but the leaflet may be retracted and quite frequently reduced to a very narrow band of tissue. Finally, annular dilation is present in approximately $95 \%$ of the cases.

Because of this complex anatomic pathology of the valve, repair may be complex and obliges to at least correction of the leaflet prolapse and remodelling and/or size reduction of the annulus (Figure 2). With regards to the latter, it is now assumed that the annular dilation may not be just functional but also caused by the disease itself.(11) In fact, the annulus loses its capacity to contract and acquires an abnormal shape which needs to be restored to 


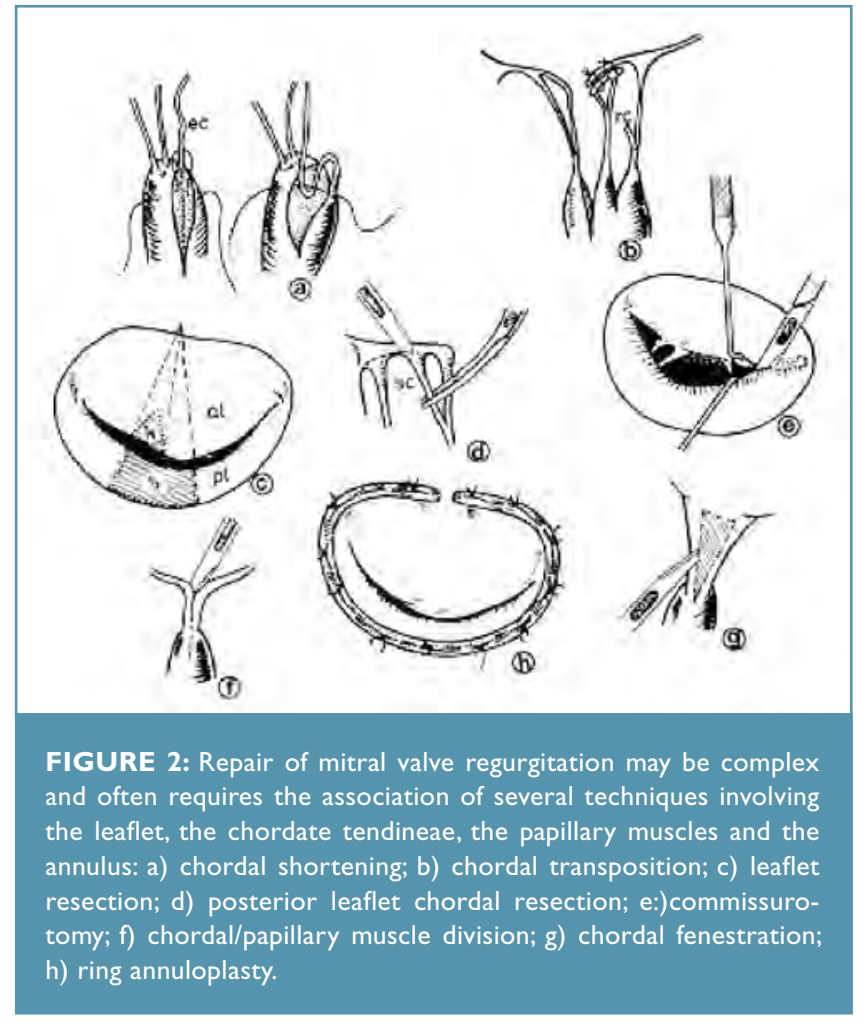

normal. Finally, dilatation of the mitral annulus was always believed to occur in its posterior segment, the anterior segment being relatively protected from enlargement, but there is recent evidence that the anterior annulus can also dilate. ${ }^{(12)}$

Correction of the anterior leaflet prolapse, as initially proposed by Carpentier, is achieved by shortening of the chordae tendineae using one or more of several techniques, of which one of the most frequent was the opening of a trench in the corresponding head of the papillary muscle and burying the excess-length segment of the chordae. ${ }^{(10)}$ This has proven to be one of weakest points of the valvuloplasty, especially in rheumatic cases, as the chordae tendineae continue to elongate due to progression of the disease. For this reason, I and others have for more than a decade now preferred the replacement/reinforcement of the chordae using artificial chordae made of GoreTex sutures (PTFE- polytetrafluorethylene). This method has proven much more durable and reproducible..$^{(13)}$

Treatment of the annular dilatation is most frequently done by implantation of an annuloplasty ring. Initially, there were two devices based on two different concepts: the rigid (Carpentier) and the flexible ring (Duran). ${ }^{(10,14)}$ Both are closed rings conferring complete protection to the annulus. Theoretically, the flexible ring should be more physiologic as it would permit the normal variation of the shape of the annulus during the cardiac cycle, as was demonstrated in the laboratory by David. ${ }^{(15)}$ However, this did not happen in practice, probably because of the pathological involvement of the annulus, as discussed above. Also, the ring becomes involved with fibrotic tissue which makes it less flexible. In my own experience, rheumatic disease requires the use of a completely rigid ring and the use of open rigid rings or bands, to protect the posterior annulus exclusively, has not yielded good results as has long ago been proven with annuloplasty consisting of sutures, as in the techniques of Reed and of Paneth. ${ }^{(16)} \mid$ believe the complete rigid rings superior in the case of rheumatic disease in contrast to what may be the norm for non-rheumatic patients.

Other techniques used in repairing rheumatic mitral valves include resection of secondary chordae of the posterior leaflet to decrease retraction and to improve the area of coaptation with the anterior leaflet. The Alfieri technique of edge-to-edge approximation may be used in extreme cases, but it was essentially developed for non-rheumatic valves with very large and mobile leaflets which can tolerate the double orifice physiology well. ${ }^{(17)}$

Importantly, the procedure must end with a perfectly competent valve. Even relatively minor degrees of residual regurgitation observed on the operating table tend to be much greater in real physiological conditions. Hence the value of intra-operative transoesophageal echocardiography, which is currently mandatory in any operation suite where these procedures are performed, is proved. Transoesophageal echocardiography has not only permitted the immediate assessment of the results, allowing correction if necessary, but also helped surgeons to better understand the anatomy and physiology of the mitral valve. 


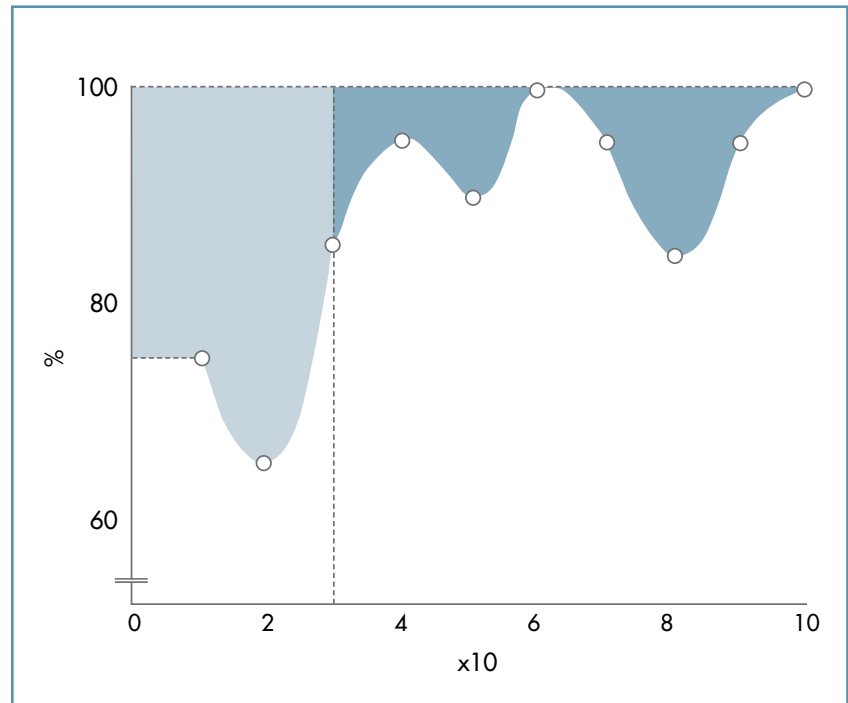

FIGURE 3: Learning curve of mitral valve repair from my own experience. Shaded areas correspond to procedure failures, by group of tens, in the first 100 patients operated on. ${ }^{(18)}$

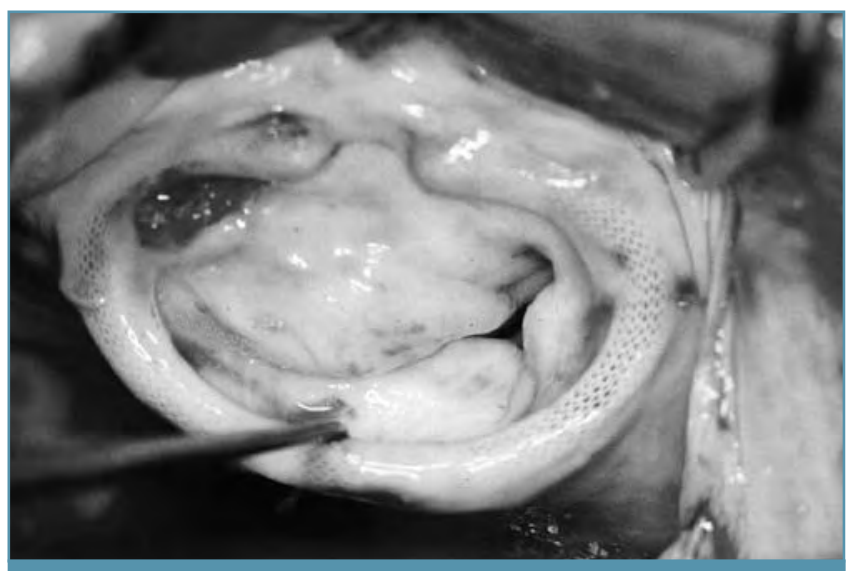

FIGURE 4: Intraoperative photograph of a mitral valve, only a few years after valvuloplasty. Fibrotic changes led to both stenosis and regurgitation, requiring valve replacement.

\section{RESULTS}

The techniques of mitral valve repair in rheumatic disease are, therefore, more complex and more difficult to master than in nonrheumatic cases, especially in the case of isolated prolapse of the mid-scallop of the posterior leaflet (P2). The resulting learning curve $^{(18)}$ (Figure 3 ) has led many surgeons to give up early in this regard. Even some experienced repair surgeons have questioned the reparability of rheumatic valves in these young patient population groups. ${ }^{(19)}$
One of the main characteristics, impacting on the results of repair of rheumatic mitral regurgitation, is that the disease is progressive and, continues even after repair. The progression of the histopathological rheumatic process inevitably leads to scarring with retraction of the leaflets and subvalvular apparatus which render the valve both stenotic and incompetent and eventually will lead to reoperation, most often to replace the valve (Figure 4). This progression of the disease, however, has significantly different consequences than those after failure of a prosthesis used for replacement. Only rarely, does the disease evolve so rapidly that it does not permit timely re-intervention. There is usually time for an adequate diagnosis and elective reoperation which, as indicated above, carries mortality similar to that of primary procedures. Hence valve-related mortality is much lower than after valve replacement. (20)

In general, the survival free from cardiac or valve-related death is in excess of $90 \%$ after five years. In my own experience in the young rheumatic population, approximately $16 \%$ of the patients required reoperation after a mean follow-up of 6 years(21) (Figure 5). In these cases, most of the valves had mixed stenosis and regurgitation and valve failure was mostly associated with the shortening of chordae, need for commissurotomy and implantation of a Carpentier ring at the initial procedure. Freedom from valve-related mortality and from valve-related complications was $96 \%$ and $80 \%$, respectively, after 6 years of follow-up. The survival complication-free was significantly worse (72\%) in patients aged 12 years or less, as compared with that observed in patients older than 12 years $(82 \%)$.

These results, observed from my own follow-up of the patients, were later confirmed in the same study-population, in a study published by Skoularigis et al., which showed a global survival of $76 \%$ and an event-free survival of $84 \%$ after a minimum of 9 years of follow-up. ${ }^{(22)}$ Sixty per cent of the patients were free from reoperation, up to ten years after the operation. Similar results have also been reported from other parts of the world. In a paper published in 2000 by Pomerantzeff et al., from S. Paulo, Brazil, a 


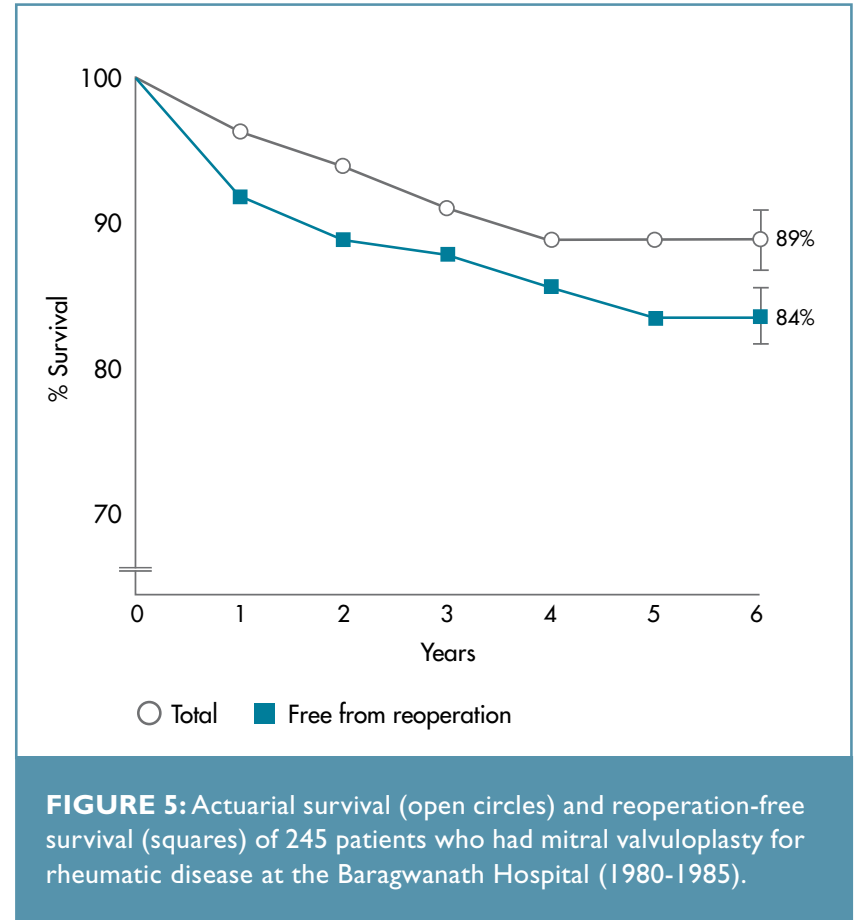

series of 201 patients with a mean age of 27 years operated on from March 1988 to December 1997 had an actual survival of 93.9\% after ten years and a survival free from reoperation of 43.3\% after the same follow-up. ${ }^{(23)}$ In 2005, Talwar et al., from India, reported on 278 children, with a mean age 1 I.7 years, who were followed for a mean of 56.5 months. The actuarial, reoperation-free and event-free survivals were $95 \%, 92 \%$ and $56 \%$, respectively. ${ }^{(24)}$

\section{RECENT EXPERIENCE WITH RHEUMATIC MITRAL} REGURGITATION IN COIMBRA

It may be interesting to compare the experience described above with my recent experience with a different population group. During the period 1988 through 2009, 464 patients with rheumatic mitral valve disease were operated on, of whom 418 had a valvuloplasty (91.1\%). The remaining 9.9\% required replacement. But it is important to note that most of the population group was constituted of patients with a mean age of 51 years, that is, patients who had rheumatic fever 3 or 4 decades ago, but a small percentage of patients coming from Africa were included. All patients younger than 20 years of age had their valve repaired.
Comparatively, during this period, mitral valve repair was possible in $94.9 \%$ of the patients with degenerative disease, in $72 \%$ of patients with infectious disease and in $93 \%$ of patients with ischaemic mitral regurgitation.

\section{CONCLUSION}

Prosthetic valve replacement in young patients carries increased morbidity and mortality. Rheumatic mitral regurgitation in this young population group is amenable to repair, although the results are less favourable than those observed with other types of mitral valve disease, especially in older populations. However, a better knowledge of the pathology and evolution of the techniques of repair have led to improved results. The latter include avoidance of resection of anterior leaflet, use of PTFE chordae versus shortening of the chordae and use of pre-shaped rigid rings. Intra-operative transoesophageal echocardiography has proven most valuable. On the other hand, better anti-failure therapy, which is mandatory in all patients, even in the presence of an excellent functional result, has improved the outcome. Naturally, continued prophylaxis of rheumatic fever remains a very important component of the treatment of these patients.

In conclusion, it is quite obvious that, even in rheumatic pathology, mitral valve repair is still worthwhile and that the percentage of valves repaired increases with the experience and the will of the surgeon to preserve the valve. In my view, mitral valve replacement is only justified when a good repair is not feasible, but the experience of the surgeon is absolutely vital. This can only be obtained by exposure to an adequate number of patients, which is usually made difficult by the political and economical situation in many underdeveloped countries. Most countries still plagued by the rheumatic epidemic, including South Africa, had the total number of cardiac operations decreased in recent years and it has been claimed that the number of patients having valve surgery is currently half of what it was some years ago. 25,26$)$ 
I. Butchart E, Gohlke-Bärwolf C, Antunes MJ, et al. Recommendations for the management of patients after heart valve surgery. Eur Heart J 2005;26:2463-7I.

2. Antunes MJ. Reoperations on cardiac valves. J Heart Valve Dis 1992; I:I5-28.

3. Davierwala PM, Borger MA, David TE, et al. Reoperation is not an independent predictor of mortality during aortic valve surgery. J Thorac Cardiovasc Surg 2006; 131:329-35

4. Antunes MJ. Prosthetic heart valve replacement. Choice of prosthesis in a young, underdeveloped population group. S Afr Med J 1985;68:755-8.

5. Antunes MJ, Vanderdonck KM, Sussman MJ. Mechanical valve replacement in children and teenagers. Eur J Cardiothorac Surg 1989;3:222-8.

6. Antunes MJ. Mitral valvuloplasty for rheumatic heart disease. Semin Thorac Cardiovasc Surg 1989;1:164-7.

7. Antunes MJ, Vieira $H$, Ferrão de Oliveira J. Open mitral commissurotomy: the 'golden standard'. J Heart Valve Dis 2000;9:472-7.

8. Vahanian A. Balloon valvuloplasty. Heart 2001;85:223-8.

9. Wang YC, Tsai FC, Chu Jj, et al. Midterm outcomes of rheumatic mitral repair versus replacement. Int Heart J. 2008;49:565-76.

10. Carpentier A. Cardiac valve surgery--the "French correction". J Thorac Cardiovasc Surg 1983;86:323-37.

I I. Barlow JB. Mitral regurgitation. In: Perspectives on the mitral valve. FA Davis Co; 1987:||3-3|.

12. Hueb AC, Jatene FB, Moreira LFP, et al. Ventricular remodelling and mitral valve modifications in dilated cardiomyopathies: new insights from anatomic study JThorac Cardiovasc Surg. 2002; 1 24:1216-24.

13. Salvador L, Mirone S, Bianuais R, et al. A 20-year experience with mitral valve repair with artificial chordae in 608 patients. J Thorac Cardiovasc Surg.2008; 135:1280-7.

14. Duran C, Luid J, Ubago M. Clinical and haemodynamic performance of a totally flexible prosthetic ring for atrioventricular valve reconstruction. Ann Thorac Surg 1976; 22:458-65.

15. David T. Effect of mitral annuloplasty ring in left ventricular function. Semin Thorac Cardiovasc Surg 1989;1:144-8.

16. Antunes MJ, Kinsley RH. Mitral valve annuloplasty: results in an underdeveloped population. Thorax 1983;38:730-6.

17. Maisano F, Torracca L, Oppizzi M, et al. The edge-to-edge technique: a simplified method to correct mitral insufficiency. Eur J Cardiothorac Surg 1998; 13:240-5.

18. Antunes MJ, Colsen PR, Kinsley RH. Mitral valvuloplasty: a learning curve. Circulation 1983;68:70-5.

19. Gometza B, al-Halees Z, Shahid M, et al. Surgery for rheumatic mitral regurgitation in patients below twenty years of age. An analysis of failures. J HeartValve Dis 1996:5:294-301.

20. Enriquez-Sarano M, Schaff HV, Orszulak TA, et al. Valve repair improves the outcome of surgery for mitral regurgitation: A multivariate analysis. Circulation 1995;91:1022-8.
21. Antunes MJ, Magalhaes MP, Colsen PR, et al. Valvuloplasty for rheumatic mitral valve disease. A surgical challenge. JThorac Cardiovasc Surg 1987;94:44-56.

22. Skoularigis J, Sinovich V, Joubert G, et al. Evaluation of the long-term results of mitral valve repair in 254 young patients with rheumatic mitral regurgitation. Circulation 1994;90:11-167-74.

23. Pomerantzeff PM, Brandao CM, Faber CM, et al. Mitral valve repair in rheumatic patients. Heart Surg Forum 2000;3:273-6.

24. Talwar S, Rajesh MR, Subramanian A, et al. Mitral valve repair in children with rheumatic heart disease. JThorac Cardiovasc Surg 2005; 129:875-9.

25. Nkomo VT. Epidemiology and prevention of valvular heart diseases and infective endocarditis in Africa. Heart. 2007;93:1510-9.

26. Commerford PJ. Valvular heart disease in South Africa in 2005. S Afr Med J 2005;95:568-74. 